TPJU-4/2001

\title{
Pion light cone wave function in the non-local NJL model
}

\author{
Michał Praszałowicz and Andrzej Rostworowski \\ M.Smoluchowski Institute of Physics, \\ Jagellonian University, \\ Reymonta 4, 30-059 Kraków, Poland.
}

(November 11, 2018)

We use the simple instanton motivated NJL-type model to calculate the leading twist pion light cone wave function. The model consists in employing the momentum dependent quark mass in the quark loop entering the definition of the wave function. The result is analytical up to a solution of a certain algebraic equation. Various properties including the $k_{T}$ dependence of the pion wave function are discussed. The resulting $k_{T}$ integrated wave function is not asymptotic and is in agreement with recent analysis of the CLEO data.

\section{INTRODUCTION}

Despite the fact that the exclusive hadron light cone wave functions were theoretically introduced 20 years ago [1]- [4], there is still relatively little experimental data which could be confronted with theory. Recently the analysis of Ref. [5] based on the latest CLEO measurements [6] put some limits on the expansion coefficients of the pion wave function in terms of the Gegenbauer polynomials. This analysis indicates that the pion wave function measured at $Q^{2}=1.5-9.2 \mathrm{GeV}^{2}$ is neither asymptotic

$$
\phi_{\pi}^{\mathrm{as}}(u)=6 u(1-u),
$$

(with $u$ being the fraction of the pion momentum carried by the quark) nor of the form proposed by Chernyak and Zhitnitsky in 1982 [7, 8]. The first attempt to study the $k_{T}$ dependence of the pion wave function has been undertaken in Refs. 99.

In order to estimate the shape of the pion wave function at sub-asymptotic $Q^{2}$, practically one theoretical approach has been used: QCD sum rules 10] which gave a variety of contradictory results [8,11]. The extension of the local sum rules to the case of the non-zero quark virtuality proposed in Refs. [12] has been recently successfully applied to model the pion wave function [13], which turned out to be in agreement with the experimental analysis of Ref. [5].

There have been also lattice calculations of the moments of the pion wave function [14,15] which, however, gave unrealistically small results. Recent transverse lattice results of Burkhardt et. al. [16] and Dalley [17] are in contradiction. Dalley's pion wave function is much more flat than the one of Ref. [16]. It is, however, not very different from our results for the constituent quark mass $M=325 \mathrm{MeV}$.

Another approach has been recently put forward in Refs. 18,19 where a simple instanton based model was applied to calculate the $u$-dependence of the pion and also photon wave function. The whole idea consisted in calculating the quark loop which enters the expression for the wave function, using the momentum dependent constituent quark mass. The momentum dependence of the quark mass appears naturally in the instanton model of the QCD vacuum [20]. It embodies the fact that the propagator of a light quark gets modified by the vacuum through which the quark propagates. The momentum dependent quark mass acts as a natural cut-off function for the $k_{T}$ integration making this integration finite. Additionally, due to the non-local $\pi$-quark coupling, it ensures vanishing of the pion wave function at the end points (see Sect.III A). If the constituent quark mass is taken as a constant and the sharp cut-off for the $k_{T}^{2}$ integration is used, the pion wave function is simply a constant. For more sophisticated cut-offs one can obtain more realistic shapes, as in Refs. 21.

The instanton model of the QCD vacuum (for recent review see e.g. 22]) allows to derive an effective nonlocal Nambu-Jona-Lasinio (NJL) type quark-meson Lagrangian where mesons are not dynamical but composite quark bilinears. By integrating out the quark fields one can, however, generate the kinetic term for the mesons. Quark-meson (pion in our case) interaction generated by the instantons is non-local and this non-locality is parameterized by the momentum dependence of the constituent quark mass. The constituent quark mass appears because of the chiral symmetry breaking induced by the instantons. However, the instanton vacuum is most probably not able to explain the confinement.

In most applications of the effective quark-meson theory described above an approximation of the constant constituent quark mass was used. This approach has an impressive success in describing baryon (which emerge as solitons) properties (see [22 and references therein). Recently an attempt to include momentum dependence in calculating solitons in the non-local NJL model has been reported in Refs. [23].

The instanton model of the QCD vacuum is naturally 
formulated in Euclidean space-time, whereas the light cone wave function is an object defined in the Minkowski space-time. One can in principle Wick rotate the underlying integrals and perform the final integrals in the Euclidean space-time as has been for example done in Refs. [24]- [26] (for early attempts to calculate the pion wave function in the instanton model see Ref. [27]). We will, however, perform all calculations directly in the Minkowski space-time. The authors of Refs. [18, 19] used a simple dipole type Ansatz for the momentum dependence of the constituent quark mass in the Minkowski space-time which made the calculations feasible. In Ref. [18] an approximation was used where the momentum dependence was taken into account only in the quarkpion vertices, whereas in Ref. 19] the calculation has been done numerically. The results of both calculations showed that the pion wave function at low momentum scale was actually not very different from the asymptotic one of Eq.(1).

In this paper we follow the approach of Refs. 118, 19, however, we show how to calculate the pion wave function analytically (up to the numerical solution of a certain algebraic equation). We obtain compact, analytical results both for $u$ and $k_{T}^{2}$ dependent wave functions. We use two parameter set of Ansätze for the momentum dependent quark mass which generalize the Ansatz of Refs. [18, 19. In this way we can study the sensitivity of various properties of the pion wave function to the specific form of the cut-off function. This is quite important, since - as explained above - we are not able to use the momentum dependent quark mass calculated in the instanton model, but we model it in such a way that the calculations can be performed in the Minkowski space-time.

A novelty of our approach is that we study the $k_{T}^{2}$ dependence of the wave function. We calculate the first moments in $k_{T}^{2}$ which are related to the mixed quarkgluon condensates [28].

Our findings can be summarized as follows. The $d^{2} k_{T}$ integrated pion wave function is not asymptotic, it exhibits a plateau for $u \sim 1 / 2$ whose height varies with the cut-off function and the constituent quark mass at zero momentum. Comparing the coefficients of the expansion in terms of the Gegenbauer polynomials, $a_{2 k}$, with the analysis of the CLEO data [5] we find that our wave function fits within $95 \%$ confidence level in the $a_{2}-a_{4}$ two dimensional parameter space, whereas the asymptotic and the Chernyak-Zhitnitsky wave functions are ruled out. One should note, however, that in the most cases the coefficient $a_{6}$ is in our case not negligible, while it was explicitly put to zero in Ref. [5]. In this respect we also differ from the model of Ref. 133.

The $k_{T}^{2}$ dependence of the $u$ integrated wave function, for $k_{T}^{2}$ below $1 \mathrm{GeV}^{2}$, is insensitive to the considered forms of the cut-off function. In this region, the wave function can be well fit by $C\left(k_{T}^{2}+\Delta^{2}\right)^{-\alpha}$ with $\alpha \sim 8 \div$ 9. For $k_{T}^{2}<0.2 \mathrm{GeV}^{2}$ the exponent fit can be used as well. The asymptotic power like behavior depends strongly upon the form of the cut-off function. However, the difference can be seen only for $k_{T}^{2}$ above few $\mathrm{GeV}^{2}$, whereas the region below $1 \mathrm{GeV}^{2}$ contributes $98 \%$ to the norm of the wave function. It indicates that the Fermilab experiment E791 9] where the typical quark (jet) $k_{T}$ is above $1 \mathrm{GeV}$, is in fact sensitive to the tiny portion of the pion wave function. The ratio $R=<k_{T}^{4}>/<k_{T}^{2}>^{2}$ is of the order of 3 indicating rather narrow $k_{T}^{2}$ distribution in contrast to Ref. [28] where $R \sim 5-7$.

We find that, given simplicity of the model, both conceptual and technical, our results are reasonable and, where the comparison with the data is possible, surprisingly accurate. The method used in this paper can be straightforwardly generalized to other wave functions like, for example, photon wave function (which we briefly discuss in Sect. [II A) or $2 \pi$ distribution amplitude.

The paper is organized as follows: in Section $\amalg$ we show how to calculate the quark loop with the momentum dependent mass analytically. The main problem here is, how to handle the complex poles of the quark propagator. In Section III] we discuss various properties of the pion wave function, like end point behavior and $k_{T}$ asymptotics. We also present numerical results and compare with other models. Finally in Section IV] we present our conclusions.

\section{PION WAVE FUNCTION WITH MOMENTUM DEPENDENT QUARK MASS}

Let us consider two quarks moving along the light cone direction $\tilde{n}=(1,0,0,1)$ parallel to the total momentum $P$ and separated by the light cone distance $z=2 \tau$ along the direction $n=(1,0,0,-1)$. The twist 2 light cone pion wave function is then defined as

$$
\begin{array}{r}
\phi_{\pi}(u)=\frac{1}{i \sqrt{2} F_{\pi}} \int_{-\infty}^{\infty} \frac{d \tau}{\pi} e^{-i \tau(2 u-1)(n P)} \\
\left\langle 0\left|\bar{\psi}(n \tau) h \gamma_{5} \psi(-n \tau)\right| \pi^{+}(P)\right\rangle
\end{array}
$$

where variable $u$, as will be shortly seen, has a meaning of a fraction of a quark momentum $k^{+}$with respect to $P^{+}$. Here $F_{\pi}=93 \mathrm{MeV}$. In this kinematical frame any four vector $v$ can be decomposed as:

$$
v^{\mu}=\frac{v^{+}}{2} \tilde{n}^{\mu}+\frac{v^{-}}{2} n^{\mu}+v_{T}^{\mu}
$$

with $v^{+}=n \cdot v, \quad v^{-}=\tilde{n} \cdot v$ and the scalar product of two four vectors reads:

$$
v \cdot w=\frac{1}{2} v^{+} w^{-}+\frac{1}{2} v^{-} w^{+}-\vec{v}_{T} \cdot \vec{w}_{T} .
$$

In Eq.(2) the path ordered exponential of the gluon field, required by the gauge invariance, has been omitted since we shall be working in the effective quark model where the gluon fields have been integrated out. 
The instanton model of the $\mathrm{QCD}$ vacuum predicts that quarks interact non-locally with an external meson field $U$ 20,22

$$
S_{I}=M \int \frac{d^{4} k d^{4} l}{(2 \pi)^{8}} \bar{\psi}(k) F(k) U^{\gamma_{5}}(k-l) F(l) \psi(l)
$$

and $U^{\gamma_{5}}(x)$ can be expanded in terms of the pion fields:

$$
U^{\gamma_{5}}=1+\frac{i}{F_{\pi}} \gamma^{5} \tau^{A} \pi^{A}-\frac{1}{2 F_{\pi}^{2}} \pi^{A} \pi^{A}+\ldots
$$

$M$ is a constituent quark mass of the order of $350 \mathrm{MeV}$ and $F(k)$ is a momentum dependent function such that $F(0)=1$ and $F\left(k^{2} \rightarrow \infty\right) \rightarrow 0$.

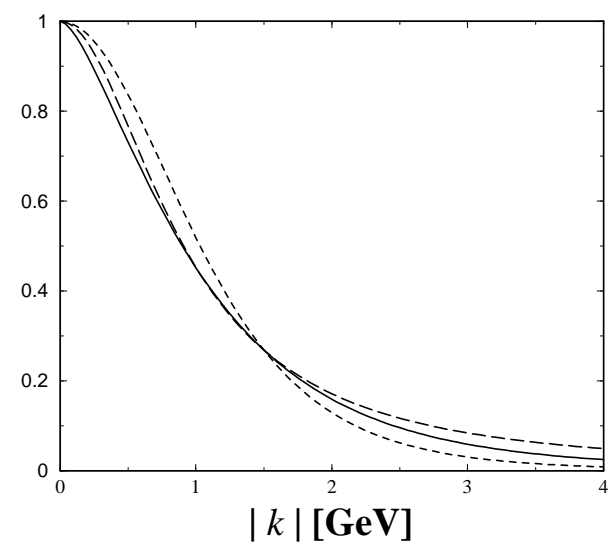

FIG. 1. $F(k)$ for Euclidean momentum $k^{2}<0$, for $n=1$ (long dashed), 3 (dashed) and for the instanton model (solid).

Function $F(k)$ has been calculated in the instanton model of the QCD vacuum

$$
F_{\text {inst. }}(k)=2 z\left[I_{0}(z) K_{1}(z)-I_{1}(z) K_{0}(z)\right]-2 I_{1}(z) K_{1}(z)
$$

where $z=k \rho / 2$. Here $1 / \rho \sim 600 \mathrm{Mev}$ is a typical inverse instanton size. One has to remember that this function is defined in Euclidean space as a Fourier transform of the instanton zero mode. Here, following Refs. [18,19] we wish to calculate $\phi_{\pi}$ directly in the Minkowski space. To this end we shall choose a simple pole formula

$M(k)=M F^{2}(k) \quad$ with $\quad F(k)=\left(\frac{-\Lambda^{2}}{k^{2}-\Lambda^{2}+i \epsilon}\right)^{n}$

which reproduces reasonably well (7) for $k^{2}<0$. This is shown in Fig.1 where we have chosen $\Lambda$ in such a way that (8) crosses with (7) for $k=1.5 \mathrm{GeV}$. Unfortunately large $k$ asymptotics $F_{\text {inst }}(k) \sim k^{-3}$ cannot be reproduced by Eq.(8) for integer $n$. Choosing half-integer $n$, however, would introduce cuts in the complex $k$ plane, making the numerical evaluation of the pion wave function unnecessary tedious. The cutoff parameter $\Lambda$ has to be chosen in such a way that the pion wave function satisfies the normalization condition

$$
\int_{0}^{1} d u \phi_{\pi}(u)=1
$$

Matrix element (2) reduces to a simple fermion loop

$$
\begin{aligned}
& \phi_{\pi}(u)=-\frac{i N_{c}}{F_{\pi}^{2} P^{+}} \int \frac{d^{4} k}{(2 \pi)^{4}} \delta\left(u-\frac{k_{+}}{P_{+}}\right) \\
& \operatorname{Tr}\left[\not h \gamma_{5} \frac{i \sqrt{M(k)}}{\not k-M(k)} \gamma_{5} \frac{i \sqrt{M(k-P)}}{(\not k-P)-M(k)}\right] .
\end{aligned}
$$

Here it is explicitly seen that $u P_{+}=k_{+}$. In the lightcone representation (3) $d^{4} k=\frac{1}{2} d k^{+} d k^{-} d^{2} \vec{k}^{\perp}$ and after performing the trace we get

$$
\begin{gathered}
\phi_{\pi}(u)=-i \frac{2 N_{c} M^{2} P_{+}}{F_{\pi}^{2}} \int \frac{d^{2} k_{T}}{(2 \pi)^{2}} \int \frac{d k_{-}}{(2 \pi)^{2}} F(k) F(k-P) \\
\frac{(1-u) F^{2}(k)+u F^{2}(k-P)}{\left[k^{2}-M^{2} F^{4}(k)+i \epsilon\right]\left[(k-P)^{2}-M^{2} F^{4}(k-P)+i \epsilon\right]}
\end{gathered}
$$

In order to calculate the integral over $d k_{-}$exactly i.e. with full $k$ dependence in the denominators we have to find zeros of the two propagators in Eq.(11). To this end it is convenient to introduce the scaled variables

$$
\begin{gathered}
p_{+}=\frac{P_{+}}{\Lambda}, \quad \kappa_{-}=\frac{k_{-}}{\Lambda}, \quad \vec{\kappa}_{T}=\frac{\vec{k}_{T}}{\Lambda} \\
\eta=p_{+} \kappa_{-}, \quad t=\left(\vec{\kappa}_{T}\right)^{2}, \quad r^{2}=\frac{M^{2}}{\Lambda^{2}} .
\end{gathered}
$$

In these variables

$$
\begin{gathered}
\phi_{\pi}(u)=-i \frac{N_{c} M^{2}}{(2 \pi)^{3} F_{\pi}^{2}} \int_{0}^{+\infty} d t \int_{-\infty}^{+\infty} d \eta \\
\frac{(1-u) \zeta_{1}^{n} \zeta_{2}^{3 n}+u \zeta_{1}^{3 n} \zeta_{2}^{n}}{G\left(\zeta_{1}\right) G\left(\zeta_{2}\right)}
\end{gathered}
$$

where

$$
\begin{aligned}
& \zeta_{1}=u\left(\eta-\frac{t+1}{u}+i \epsilon \operatorname{sign}(u)\right) \\
& \zeta_{2}=-(1-u)\left(\eta+\frac{t+1}{(1-u)}-i \epsilon \operatorname{sign}(1-u)\right)
\end{aligned}
$$

and

$$
G(z)=z^{4 n+1}+z^{4 n}-r^{2}=\prod_{i=1}^{4 n+1}\left(z-z_{i}\right)
$$

with $z_{i}$ being $(4 n+1)$ roots of the equation

$$
G(z)=0 .
$$


We aim at evaluating the $d \eta$ integral by contour integration. The $2(4 n+1)$ simple poles of the integrand (13) lie at

$$
\begin{aligned}
& \eta_{i}^{(1)}=\frac{t+1+z_{i}}{u}-i \epsilon \operatorname{sign}(u), \\
& \eta_{i}^{(2)}=-\frac{t+1+z_{i}}{1-u}+i \epsilon \operatorname{sign}(1-u) .
\end{aligned}
$$

Let's assume for definiteness that $0 \leq u \leq 1$. For $r^{2}=$ $0\left(\Lambda^{2}=\infty\right)$ the roots of Eq. (16) are $z_{1}=\ldots=z_{4 n}=0$, $z_{4 n+1}=-1$ so the poles $\eta^{(1)}$ lie below and the poles $\eta^{(2)}$ lie above the Re $\eta$ axis. When $r^{2}$ increases $\left(\Lambda^{2}\right.$ decreases from $\infty$ to its physical value) the roots $z_{i}$ drift in the complex z-plane as shown in Fig. 2. Therefore some of the poles $\eta_{i}^{(1)}$ can move above and some of the poles $\eta_{i}^{(2)}$ can move below the Re $\eta$ axis. The crucial point is that if we kept the integration path going along the Re $\eta$ axis we would get an unphysical result: $\phi_{\pi}(u)$ would not vanish outside the interval $0 \leq u \leq 1$. We assume that physically correct prescription for performing the $d \eta$ integral is to deform the integration path as shown in Fig. 3.

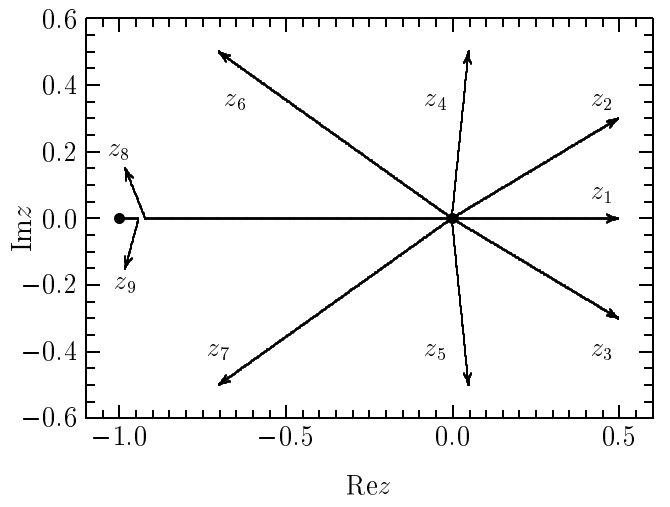

FIG. 2. Flow of the zeros of $G(z)$ with increasing $r$ for $n=2$. For $r=0(\Lambda=\infty)$ there are $4 n$ degenerate solutions $z_{1}, \ldots, z_{4 n}$ and one non degenerate solution $z_{4 n+1}=-1$. With increasing $r$ ( $\Lambda$ decreasing from $\infty$ to the physical value) $z_{4 n+1}$ solution drifts rightwards toward 0 , and $4 n$ zero solutions split into 2 real ones, $z_{4 n}$ drifting towards $z_{4 n+1}$ and $z_{1}$ towards $+\infty$, and $4 n-2$ complex (pairwise conjugated) solutions $z_{2 i}, z_{2 i+1}, i=1, \ldots, 2 n-1$ which flow into the upper and lower parts of the complex $z$ plane. When $r$ reaches the value of the local maximum $r_{\max }$ of $G(z)$ for $-1<z<0$ the two real zeros $z_{4 n}$ and $z_{4 n+1}$ meet and "scatter" drifting further into the upper $\left(z_{4 n}\right)$ and lower $\left(z_{4 n+1}\right)$ half of the complex $z$ plane.

Closing the contour in the upper complex $\eta$ half-plane we should enclose all the poles $\eta_{i}^{(2)}$ and none of the poles $\eta_{i}^{(1)}$. This prescription ensures that $\phi_{\pi}(u)$ is real and vanishes outside the interval $0 \leq u \leq 1$. Now, the $d \eta$ integral yields

$$
\begin{array}{r}
\phi_{\pi}(u)=\frac{N_{c} M^{2}}{(2 \pi)^{2} F_{\pi}^{2}}(-1+u)^{n} \int_{0}^{+\infty} d t \sum_{i=1}^{4 n+1} z_{i}^{n} f_{i} \\
\frac{u\left(t+1+u z_{i}\right)^{3 n}+(1-u)^{2 n+1} z_{i}^{2 n}\left(t+1+u z_{i}\right)^{n}}{\prod_{k=1}^{4 n+1}\left[t+1+u z_{i}+(1-u) z_{k}\right]}
\end{array}
$$

where factors $f_{i}$ are defined as

$$
f_{i}=\prod_{\substack{k=1 \\ k \neq i}}^{4 n+1} \frac{1}{z_{k}-z_{i}} .
$$

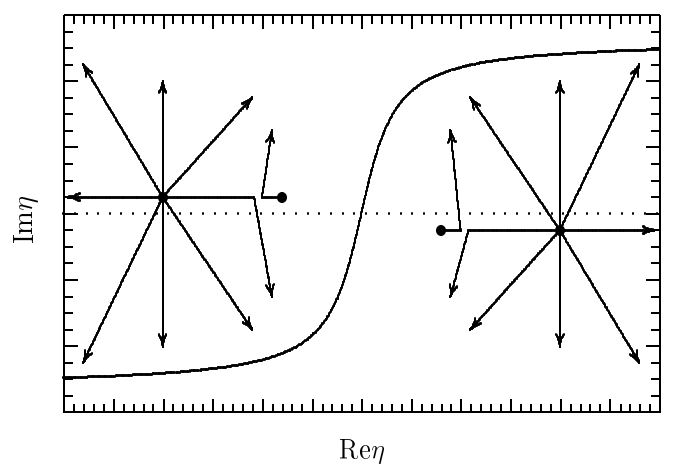

FIG. 3. Schematic flow of the $\eta$ poles with increasing $r$ for $n=2$ together with the integration contour in the complex $\eta$ plane. For $r=0$ the poles are shown as black dots and the integration contour is represented by the dotted line.

With the help of the following identity

$$
\begin{aligned}
& \frac{u\left(t+1+u z_{i}\right)^{3 n}+(1-u)^{2 n+1} z_{i}^{2 n}\left(t+1+u z_{i}\right)^{n}}{\prod_{k=1}^{4 n+1}\left[t+1+u z_{i}+(1-u) z_{k}\right]} \\
& =\frac{1}{(-1+u)^{n}} \sum_{k=1}^{4 n+1} z_{k}^{n} f_{k} \frac{u z_{k}^{2 n}+(1-u) z_{i}^{2 n}}{t+1+u z_{i}+(1-u) z_{k}}
\end{aligned}
$$

the result for the $k_{T}^{2}$-dependent pion wave function $\Phi\left(u, k_{T}^{2}\right)$ can be cast in the following compact form:

$$
\begin{aligned}
\Phi_{\pi}\left(u, k_{T}^{2}\right) & =\frac{1}{\Lambda^{2}} \frac{N_{c} M^{2}}{(2 \pi)^{2} F_{\pi}^{2}} \\
& \sum_{i, k=1}^{4 n+1} f_{i} f_{k} \frac{z_{i}^{n} z_{k}^{3 n} u+z_{i}^{3 n} z_{k}^{n}(1-u)}{\frac{k_{T}^{2}}{\Lambda^{2}}+1+z_{i} u+z_{k}(1-u)} .
\end{aligned}
$$

Factors $f_{i}$ obey the following properties which are crucial for the finiteness of the $d\left(k_{T}^{2}\right)$ integration and for the end point behavior of the pion (and also photon) wave function

$$
\sum_{i=1}^{4 n+1} z_{i}^{m} f_{i}=\left\{\begin{array}{l}
0 \text { for } m<4 n \\
1 \text { for } m=4 n
\end{array}\right.
$$


It is interesting to note that properties 22 hold for any set of $N=4 n+1$ numbers, irrespectively of the fact that they are solutions of certain equation of degree $N$.

Due to Eq. 222 $\Phi_{\pi}\left(u, k_{T}^{2}\right)$ vanishes for large $k_{T}^{2}$ and the $d\left(k_{T}^{2}\right)$ integration is finite so that

$$
\begin{aligned}
\phi_{\pi}(u) & =\int_{0}^{\infty} d\left(k_{T}^{2}\right) \Phi_{\pi}\left(u, k_{T}^{2}\right) \\
& =-\frac{N_{c} M^{2}}{(2 \pi)^{2} F_{\pi}^{2}} \sum_{i, k} f_{i} f_{k}\left(z_{i}^{n} z_{k}^{3 n} u+z_{i}^{3 n} z_{k}^{n}(1-u)\right) \\
& \times \ln \left(1+z_{i} u+z_{k}(1-u)\right) .
\end{aligned}
$$

Eqs.(21,23) are our final results for the pion light cone wave function for the momentum dependent quark mass (8). These analytical formulae depend on the numerical solutions $z_{i}$ of Eq.(16). Cutoff parameter $\Lambda$ can be found by imposing the normalization condition (9).

\section{PROPERTIES OF THE PION WAVE FUNCTION}

\section{A. Analytical properties}

Function $\phi_{\pi}(u)=\phi_{\pi}(1-u)$ which is a trivial property of the symmetry of exchanging two sums over $i$ and over $k$ in Eq.(23). At the end points $\phi_{\pi}(u)$ vanishes. Indeed

$$
\phi_{\pi}(0)=-\frac{N_{c} M^{2}}{(2 \pi)^{2} F_{\pi}^{2}} \sum_{i} f_{i} z_{i}^{3 n} \sum_{k} f_{k} z_{k}^{n} \ln \left(1+z_{k}\right)=0
$$

as a consequence of Eq.(22). In fact for small $u \phi_{\pi}(u) \sim$ $u^{n}$. The reason for this kind of behavior is the presence of the factor $\sqrt{M(k) M(k-P)}$ in Eq. 10). Indeed, one can calculate in the same way the transverse photon wave function $\phi_{\gamma}^{\perp}(u)$ which, since the $\gamma$-quark coupling is local, does not contain the dumping factor $\sqrt{M(k) M(k-P)}$. The result reads

$$
\begin{aligned}
\phi_{\gamma}^{\perp}(u) & =-\frac{N_{c} M}{(2 \pi)^{2} f_{\gamma}^{\perp}} \sum_{i, k} f_{i} f_{k}\left(z_{i}^{2 n} z_{k}^{4 n} u+z_{i}^{4 n} z_{k}^{2 n}(1-u)\right) \\
& \times \ln \left(1+z_{i} u+z_{k}(1-u)\right)
\end{aligned}
$$

and the wave function $\phi_{\gamma}^{\perp}(0)=\phi_{\gamma}^{\perp}(1) \neq 0$, due to the second property (22). So despite of the nonperturbative effects embodied in the momentum dependent quark mass (8) the point like photon-quark coupling makes the photon wave function non vanishing at the end points.

The advantage of the present calculation is that we can calculate not only the $u$ dependence, but also the $k_{T}^{2}$ dependence of the pion wave function. Integrating $\Phi_{\pi}\left(u, k_{T}^{2}\right)$ over $u$ we get

$$
\tilde{\phi}_{\pi}\left(k_{T}^{2}\right)=\int_{0}^{1} d u \Phi_{\pi}\left(u, k_{T}^{2}\right) .
$$
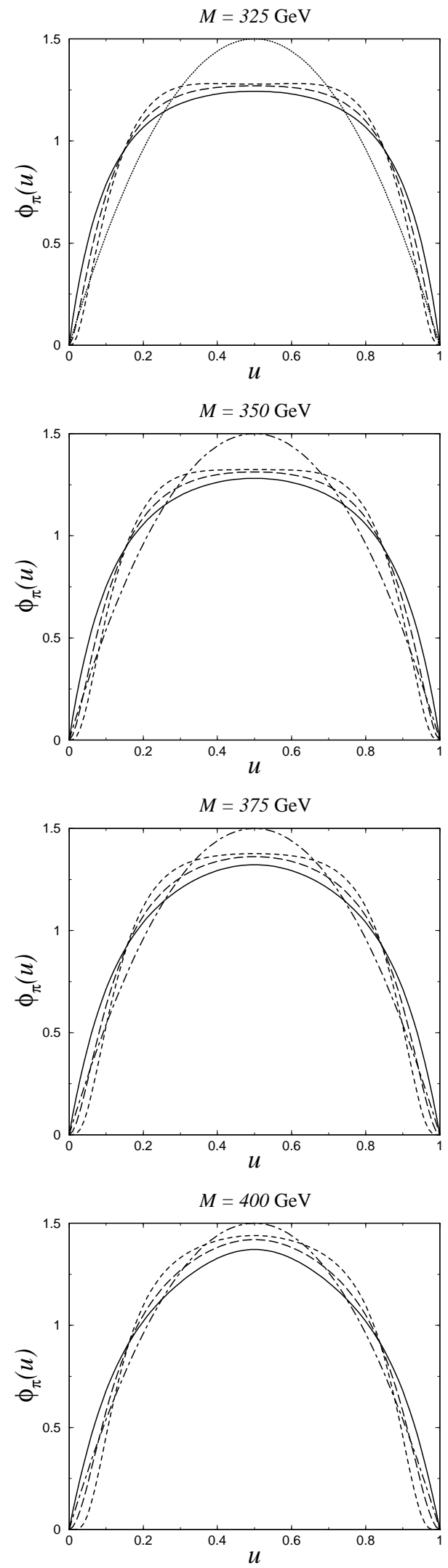

FIG. 4. Pion wave function for $M=325,350,375$ and $400 \mathrm{MeV}$, for $n=1$ (solid), 2 (long dashed) and 5 (dashed) together with the asymptotic wave function (dot-dashed). 
By expanding $\tilde{\phi}\left(k_{T}^{2}\right)$, Eq. 26), for large $k_{T}^{2}$ we find

$$
\tilde{\phi}_{\pi}\left(k_{T}^{2}\right) \sim\left(\frac{\Lambda^{2}}{k_{T}^{2}}\right)^{4 n+1} .
$$

This kind of behavior differs from the commonly assumed exponential form of $\tilde{\phi}_{\pi}\left(k_{T}^{2}\right)$. Let us, however, remark that the asymptotic behavior (27) which switches on for $k_{T}^{2}>\Lambda^{2}$ concerns only a negligible tail of the whole wave function. Moreover, the power in Eq.(27) is large enough, even for $n=1$, to make the predictions of the first $k_{T}^{2}$ moments reliable. We shall come back to this point in the next Section.

\section{B. Numerical results}

In Fig. 1 we plot $\phi_{\pi}(u)$ for $M=325,350,375,400 \mathrm{MeV}$ and $n=1,2$ and 5 . In fact for $n>5 \phi_{\pi}(u)$ does not change much anymore. It is clearly seen from these figures that in all cases the NJL model with the nonlocal regulator (8) gives $\phi_{\pi}(u)$ which is different from the asymptotic one. This difference is more pronounced for smaller masses $M$ and larger $n$ 's. For $M=325$ and $350 \mathrm{Mev}$ and $n=5$ a shallow minimum can be seen at $u=0.5$.

It is instructive to compare our result with the one of Refs. 18, 19 where the momentum dependence of the quark mass has been taken into account only in the numerators of Eq.(11). This is done in Fig. 5 for $M=350$ $\mathrm{MeV}$ and $n=1$. It is clearly seen that the momentum dependence of the denominators flattens the pion wave function making it "less asymptotic".

TABLE I. Gegenbauer coefficients

\begin{tabular}{cccrrrr}
\hline \hline $\begin{array}{c}M \\
\mathrm{MeV}\end{array}$ & $\mathrm{n}$ & $\begin{array}{c}\Lambda \\
\mathrm{MeV}\end{array}$ & $a_{2}$ & $a_{4}$ & $a_{6}$ & $a_{8}$ \\
\hline \multirow{3}{*}{325} & 1 & 1249 & 0.1363 & 0.0215 & 0.0037 & 0.0007 \\
& 2 & 1862 & 0.0935 & -0.0183 & -0.0155 & -0.0077 \\
& 5 & 3033 & 0.0606 & -0.0493 & -0.0217 & -0.0060 \\
\hline \multirow{3}{*}{350} & 1 & 1156 & 0.1144 & 0.0151 & 0.0015 & 0.0001 \\
& 2 & 1727 & 0.0659 & -0.0271 & -0.0166 & -0.0064 \\
& 5 & 2819 & 0.0284 & -0.0600 & -0.0202 & -0.0020 \\
\hline \multirow{3}{*}{375} & 1 & 1081 & 0.0931 & 0.0113 & -0.0003 & -0.0002 \\
& 2 & 1621 & 0.0387 & -0.0325 & -0.0166 & -0.0047 \\
& 5 & 2649 & -0.0035 & -0.0668 & -0.0173 & 0.0025 \\
\hline \multirow{3}{*}{400} & 1 & 1020 & 0.0723 & 0.0099 & -0.0020 & -0.0001 \\
& 2 & 1534 & 0.0118 & -0.0347 & -0.0164 & -0.0027 \\
& 5 & 2512 & -0.0351 & -0.0695 & -0.0138 & 0.0072 \\
\hline \hline
\end{tabular}

\footnotetext{
${ }^{1}$ The exponential fit, however, is quite satisfactory for $k_{T}^{2}<$ $0.2 \mathrm{GeV}^{2}$.
}

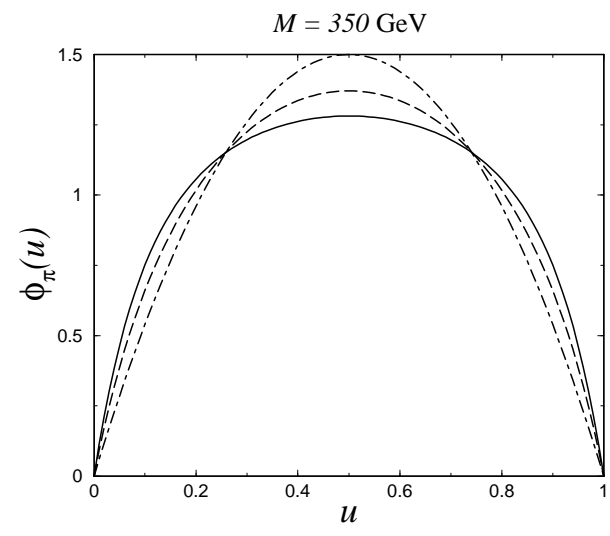

FIG. 5. Pion wave function for $M=350 \mathrm{MeV}$, for $n=1$ (solid), compared with the approximate result of Ref.[17] (dashed) and the asymptotic wave function (dot-dashed).

The comparison of different wave functions and especially their content of the non-asymptotic terms is readily done by expanding $\phi_{\pi}(u)$ in terms of the Gegenbauer polynomials:

$$
\begin{aligned}
\phi_{\pi}(u) & =\phi_{\pi}^{\mathrm{as}}(u)\left[1+a_{2} C_{2}^{3 / 2}(2 u-1)+a_{4} C_{4}^{3 / 2}(2 u-1)\right. \\
& \left.+a_{6} C_{6}^{3 / 2}(2 u-1)+\ldots\right]
\end{aligned}
$$

where the coefficients $a_{2 k}$ depend on the scale $\mu^{2}$. In model calculations it is, however, hard to define precisely what value should be taken for $\mu$. Clearly $\mu$ is of the order of the cutoff $\Lambda$. In Table 1 we show the cutoff $\Lambda$ and $a_{2}, \ldots, a_{8}$ for $M=325,350,375,400 \mathrm{MeV}$ and $n=1,2$ and 5 . It is clearly seen that in most cases $a_{6}$ is still comparable with $a_{4}$. Only $a_{8}$ is always substantially smaller than $a_{2}, a_{4}$ or $a_{6}$. Interestingly for $M=325 \mathrm{MeV}$ and $n=1$ we get $a_{2}$ very similar to the transverse lattice result of Dalley [17] who gets $a_{2}^{\text {latt. }}=0.133$.

As already stated in the previous sections the non-local NJL model gives the pion wave function as a function of two variables $u$ and $k_{T}^{2}$. In Fig. 6 we plot $\tilde{\phi}_{\pi}\left(k_{T}^{2}\right)$ defined in Eq.(26) for $M=350 \mathrm{Mev}$ and $n=1,2$ and 5. These functions are indistinguishable within the accuracy of the plot. $\tilde{\phi}_{\pi}\left(k_{T}^{2}\right)$ can be well approximated by the function $C\left(k_{T}^{2}+\Delta^{2}\right)^{-\alpha}$, with $\alpha=9, \Delta=1 \mathrm{GeV}^{2}$ and $C=8$ $\mathrm{GeV}^{16}$. The $n$ dependence is visible only for $k_{T}^{2}$ above a few $\mathrm{GeV}^{2}$ and is not taken into account in this fit.

In order to illustrate the (un)importance of the asymptotic power like behavior, let us define

$$
\mathcal{N}(\lambda)=\int_{0}^{\lambda^{2}} d\left(k_{T}^{2}\right) \tilde{\phi}_{\pi}\left(k_{T}^{2}\right) .
$$

Obviously $\mathcal{N}(\infty)=1$. However, $\mathcal{N}(\lambda)$ saturates relatively fast

$$
\mathcal{N}(1 \mathrm{GeV}) \sim 0.98
$$


for all masses and powers of $n$. In fact the higher $n$ the larger value of $\mathcal{N}(1 \mathrm{GeV})$. This observation indicates that the bulk of the wave function comes from the region where there is little, if any, dependence on the cutoff parameter $n$. Outside this region the $k_{T}^{2}$-dependent wave function starts to depend strongly on the form of the cutoff function (8). Unfortunately the $k_{T}$ range in the recently measured diffractive dijet production in the pion nucleus scattering [9] is precisely in this asymptotic regime. Therefore we have to conclude that the information provided by this piece of data cannot be used to test our model, unless the $k_{T}$ range is not shifted towards the $\mathrm{MeV}$ rather than $\mathrm{GeV}$ region.

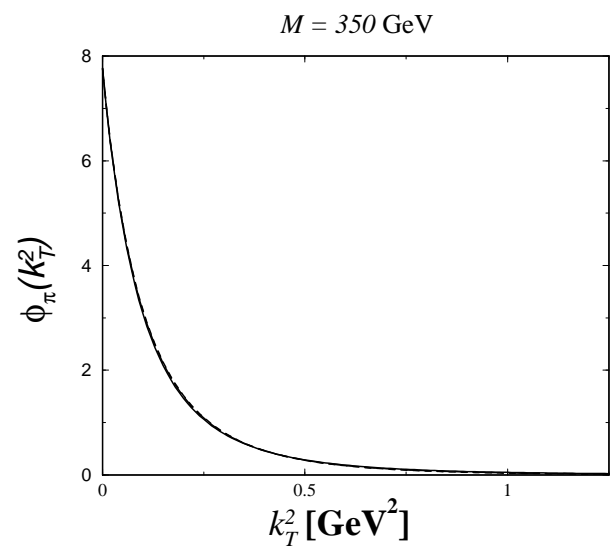

FIG. 6. $\tilde{\phi}_{\pi}\left(k_{T}^{2}\right)$ for $M=350$ and $n=1,2$ and 5 . These curves are indistinguishable within the scale of the plot.

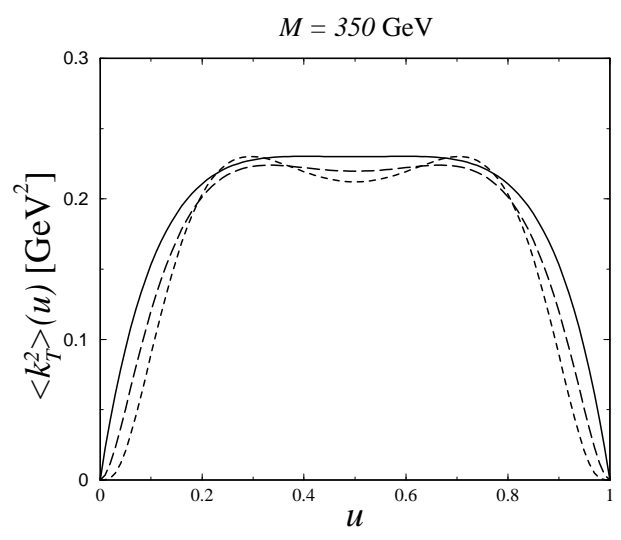

FIG. 7. $<k_{T}^{2}>$ in pion wave function for $M=350 \mathrm{MeV}$, and $n=1$ (solid), 2 (long dashed) and 5 (dashed).

In order to see how the transverse momentum is distributed among quarks with different longitudinal momentum it is instructive to plot $\left\langle k_{T}^{2}>\right.$ as a function of $u$. This is depicted in Fig. [. One can see that $<k_{T}^{2}>$ saturates relatively fast and remains almost constant for $0.2<u<0.8$. The saturation value decreases with an increasing $M$. In Table II average $\left\langle k_{T}^{2}>\right.$ and $\left\langle k_{T}^{4}>\right.$ are displayed together with the ratio $R=<k_{T}^{2}>^{2} /<k_{T}^{4}>$.
It was argued in Ref. 28 that the QCD sum rules predict $R \sim 4-5$. In our case $R$ is almost two times smaller, however the absolute value of $\left\langle k_{T}^{2}\right\rangle$ is slightly larger than the one of Ref. 28]. Let us remind that the $k_{T}^{2}$ moments are related to the mixed quark-gluon condensates which are poorly known in QCD.

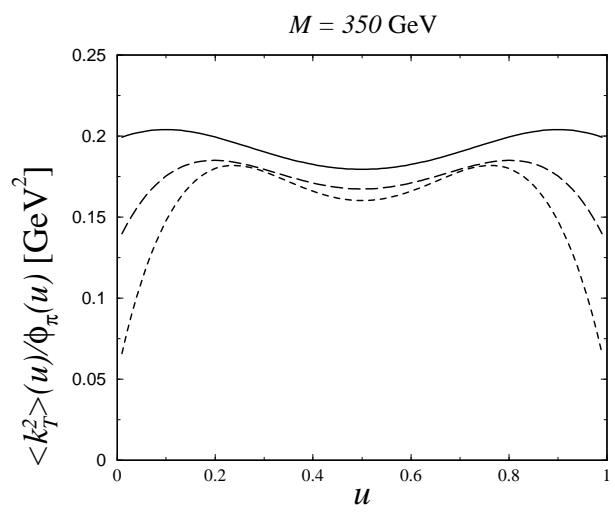

FIG. 8. Ratio $<k_{T}^{2}>(u) / \phi_{\pi}(u)$ for $M=350 \mathrm{MeV}$, and $n=1$ (solid), 2 (long dashed) and 5 (dashed). If there was factorization, this ratio should not depend upon $u$.

We have checked the hypothesis that $\Phi\left(u, k_{T}^{2}\right)$ factorizes:

$$
\Phi_{\pi}\left(u, k_{T}^{2}\right)=\phi_{\pi}(u) \tilde{\phi}_{\pi}\left(k_{T}^{2}\right)
$$

If this was the case we should get $\left\langle k_{T}^{2}\right\rangle(u) / \phi_{\pi}(u)=$ const $=<k_{T}^{2}>$. The actual plot of $<k_{T}^{2}>(u) / \phi_{\pi}(u)$ dependence is presented in Fig.8.

\section{Comparison with the data}

Expansion of the pion wave function in terms of the Gegenbauer polynomials has been defined in Eq. (28) and the values of the expansion coefficients for $\phi_{\pi}(u)$ of Eq.(23) are displayed in Table II. Schmedding and

TABLE II. The values of $<k_{T}^{2}>,<k_{T}^{4}>$ and $\frac{<k_{T}^{4}>}{<k_{T}^{2}>^{2}}$

\begin{tabular}{|c|c|c|c|c|}
\hline $\begin{array}{c}M \\
\mathrm{MeV}\end{array}$ & $\mathrm{n}$ & $\begin{array}{c}<k_{T}^{2}> \\
\mathrm{GeV}^{2}\end{array}$ & $\begin{array}{c}<k_{T}^{4}> \\
\mathrm{GeV}^{4}\end{array}$ & $\left\langle k_{T}^{4}>/<k_{T}^{2}>^{2}\right.$ \\
\hline \multirow{3}{*}{325} & 1 & $(0.451)^{2}=0.203$ & $(0.620)^{4}=0.148$ & 3.58 \\
\hline & 2 & $(0.433)^{2}=0.188$ & $(0.564)^{4}=0.101$ & 2.90 \\
\hline & 5 & $(0.424)^{2}=0.180$ & $(0.542)^{4}=0.086$ & 2.67 \\
\hline \multirow{3}{*}{350} & 1 & $(0.437)^{2}=0.191$ & $(0.593)^{4}=0.124$ & 3.39 \\
\hline & 2 & $(0.420)^{2}=0.176$ & $(0.541)^{4}=0.086$ & 2.76 \\
\hline & 5 & $(0.411)^{2}=0.169$ & $(0.520)^{4}=0.073$ & 2.55 \\
\hline \multirow{3}{*}{375} & 1 & $(0.426)^{2}=0.182$ & $(0.572)^{4}=0.107$ & 3.24 \\
\hline & 2 & $(0.409)^{2}=0.167$ & $(0.522)^{4}=0.074$ & 2.65 \\
\hline & 5 & $(0.402)^{2}=0.162$ & $(0.503)^{4}=0.064$ & 2.45 \\
\hline \multirow{3}{*}{400} & 1 & $(0.418)^{2}=0.175$ & $(0.555)^{4}=0.095$ & 3.11 \\
\hline & 2 & $(0.402)^{2}=0.162$ & $(0.508)^{4}=0.067$ & 2.55 \\
\hline & 5 & $(0.394)^{2}=0.155$ & $(0.489)^{4}=0.057$ & 2.37 \\
\hline
\end{tabular}


Yakovlev [5] performed recently a theoretical analysis of the latest CLEO [6] data on pion electroproduction from a quasi real photon. They constructed a $95 \%$ and $68 \%$ confidence level contour plots in the $a_{2}-a_{4}$ parameter space for an average virtuality $\mu=2.4 \mathrm{GeV}$. In Fig.9 we present their contours (Fig.6 in [5]) together with our values of $a_{2}$ and $a_{4}$ for various fits displayed in Table If. We have not performed the QCD evolution of these coefficients since it is not clear which scale should be taken as a starting point $\mu_{0}$ of the evolution. It is naturally to assume that $\mu_{0} \sim \Lambda$. In that case, however, the evolution would be very weak, since - depending on the cut-off function $-\Lambda$ changes between 1 and $3 \mathrm{GeV}$. As the result of the evolution the points in the $a_{2}-a_{4}$ plane would slightly move towards the asymptotic wave function.

Let us finally note, that the analysis of Ref. [5] has been performed under the assumption that $a_{6}=0$. As can be seen from Table for $n>1 a_{6}$ is still comparable with $a_{4}$.

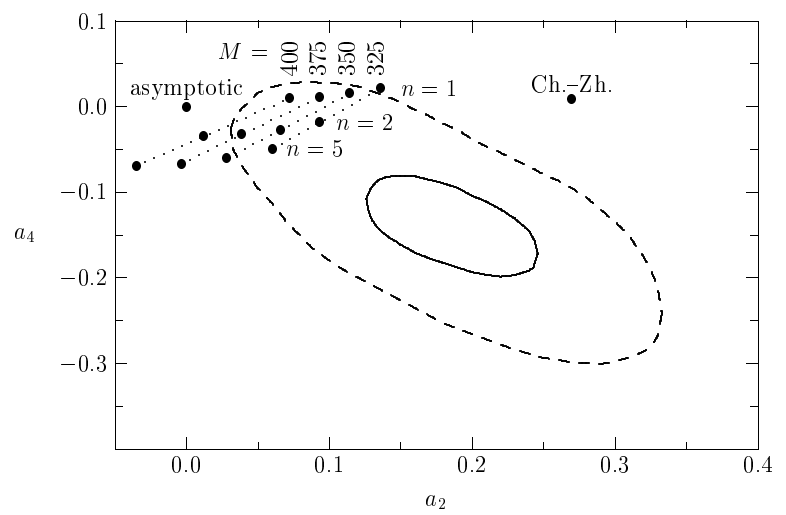

FIG. 9. The parameter space $\left(a_{2}, a_{4}\right)$ of Ref.[5]. Black dots represent different model predictions, solid contour corresponds to $68 \%$ confidence level, whereas the dashed one to $95 \%$.

\section{SUMMARY AND CONCLUSIONS}

In this paper we have calculated both $u$ and $k_{T}^{2}$ dependence of the light cone twist 2 pion wave function in the non-local NJL model. The nonperturbative nature of this approach is embodied in the momentum dependence of the constituent quark mass. In principle this dependence is known from the instanton model of the QCD vacuum. However, in order to be able to carry out the calculations in the Minkowski space-time we have used a class of simple Ansätze (8), which for Euclidean momenta relatively well reproduce $M_{\text {inst. }}(k)$. In this way we have constructed a transparent and tractable model which allowed to calculate the pion wave function $\phi_{\pi}(u)$ analytically. We have shown how for the finite cut-off parameter $\Lambda$ the Feynman integration contour has to be modified in order that $\phi_{\pi}(u)$ is real and vanishes outside $0<u<1$.

Our phenomenological results are encouraging. For the constituent masses at zero momentum between $M=$ $325-350 \mathrm{MeV}$ and $n=2$ (see Eq.(8)) our results nicely fit into the $95 \%$ confidence region of Ref. [5]. In comparison with the asymptotic wave function (11) and with the results of Refs. 18. 19] where the momentum dependence of the constituent mass in the denominator (c.f. Eq. (11) was neglected, our wave function is broader with, however, less pronounced end point regions. Similar behavior was found in Ref. [13] where not only non-locality (within the sum rules approach) but also radiative corrections have been taken into account. The fact that the true pion distribution amplitude may be broader than the asymptotic one has been already pointed out in Ref. 29.

Although at first sight we have found rather strong sensitivity to the power $n$ in the $k_{T}^{2}$ distribution, it turned out that the power like asymptotic tail $\tilde{\phi}_{\pi}\left(k_{T}^{2}\right) \sim$ $\left(1 / k_{T}^{2}\right)^{4 n+1}$ switches on at rather high momenta of the order of a few $\mathrm{GeV}$. For small and moderate $k_{T}^{2}$ there is no dependence of $\tilde{\phi}_{\pi}\left(k_{T}^{2}\right)$ on $n$. We have shown that more than $98 \%$ of the norm of $\tilde{\phi}_{\pi}\left(k_{T}^{2}\right)$ is concentrated in the region of $k_{T}^{2}<1 \mathrm{GeV}^{2}$. Therefore recent measurements of the diffractive dissociation of a pion into 2 jets with transverse momentum above $1 \mathrm{GeV}$ [9] test the tiny portion of the wave function. Most probably, since the higher $k_{T}^{2}$ moments are divergent for the power like tail, this region is not reliably described by our model.

Another way to extract a light cone wave function would be to calculate the entire physical process within the effective model, rather than only the wave function itself as given by Eq.(2). This approach has been advocated recently in Ref. [26] and for the process $\gamma \gamma \rightarrow \pi$ can be in principle carried out also within our approach. It would be interesting to see what is the difference between the wave function calculated directly from Eq.(2) and according to the prescription of Ref. [26].

As a by-product we have also calculated the transverse photon wave function $\phi_{\gamma}^{\perp}(u)$ which is almost insensitive to the parameter $n$ of the constituent quark mass. Further application of our method to strange mesons and, for example, $2 \pi$ distribution amplitudes is straightforward and will be discussed elsewhere. It is also possible by calculating pion wave functions with other Dirac structures in (2) to disentangle different condensates related to the $k_{T}^{2}$ moments [28].

This work was partially supported by the Polish KBN Grant PB 2 P03B 019 17. M.P. is grateful to W.Broniowski, K.Goeke, P.V.Pobylitsa, M.V.Polyakov and N.G.Stefanis for discussions and interesting suggestions. The authors kindly acknowledge comments and remarks of A.Bakulev, A.Dorokhov and T.Heinzl. 
[1] V.L. Chernyak and A.R. Zhitnitsky, JETP Lett. 26 (1977) 359.

[2] S. Brodsky and G.P. Lepage, Phys. Lett. B87 (1979) 594; Phys. Rev D22 (1980) 2157.

[3] G. Farrar and D. Jackson, Phys. Rev. Lett. 43 (1979) 246.

[4] A.V. Efremov and A.V. Radyushkin, Theor. Mat. Phys. 42 (1980) 97; Phys. Lett. B94 (1980) 245.

[5] A. Schmedding and O. Yakovlev, Phys. Rev D62 (2000) 116002, hep-ph/9905392.

[6] J. Gronberg (CLEO Collaboration), Phys. Rev D57 (1998) 33, hep-ex/9707031.

[7] V. L.Chernyak and A. R. Zhitnitsky, Nucl. Phys. B201 (1982) 492; ibid B214 (1983) 547(E).

[8] V.L. Chernyak and A.R. Zhitnitsky, Phys. Rep. 112 (1984) 173.

[9] E.M. Aitala et.al. (Fermilab E791 Collaboration), hepex/0010043; D. Ashery, hep-ex/99100024.

[10] M.A. Shifman, A.I. Vainshtein and V.I. Zakharov, Nucl. Phys. B147 (1979) 385.

[11] V.M. Braun and I.E. Filyanov, Z. Phys. C44 (1989) 157.

[12] S.V. Mikhailov and A.V. Radyushkin, JETP Lett. 43 (1986) 712; Sov. J. Nucl. Phys. 49 (1989) 494; Phys. Rev. D45 (1992) 1754.

[13] A.P. Bakulev, S.V. Mikhailov and N.G. Stefanis, Ruhr-University preprint 2001, RUB-TPII-03/01, hep$\mathrm{ph} / 0103119$.

[14] D. Daniel, R. Gupta and D.G. Richards, Phys. Rev. D43 (1991) 3715.

[15] L. Del Debbio et. al., Nucl. Phys. Proc. Suppl. 83 (2000) 235, hep-lat/9909147.

[16] M. Burkardt and H. El-Khozondar, Phys. Rev. D60 (1999) 054504, hep-ph/9805495; M. Burkardt and S. Seal, hep-ph/0101338.

[17] S. Dalley, hep-ph/0010043.

[18] V.Yu. Petrov and P.V. Pobylitsa, hep-ph/9712203.

[19] V.Yu. Petrov, M.V. Polyakov, R. Ruskov, C. Weiss and K. Goeke, Phys. Rev. D59 (1999) 114018, hepph/9807229.

[20] D.I. Diakonov and V.Yu. Petrov, Nucl. Phys. B245 (1984) 259; B272 (1986) 457.

[21] T. Heinzl, Ligh-Cone Quantization: Foundations and Applications, hep-th/0008096; Nucl. Phys. Proc. Suppl. 90 (2000) 83, hep-ph/0008314.

[22] D.I. Diakonov and V.Yu. Petrov, hep-ph/0009006.

[23] B. Golli, W. Broniowski and G. Ripka, Phys. Lett. B 437 (1998) 24, hep-ph/9807261; G. Ripka and B. Golli, hep-ph/9910479.

[24] S.V. Esaibegyan, S.N. Tamarian, Sov. J. Nucl. Phys. 51 (1990) 310 [Yad. Fiz. 51 (1990) 485].

[25] A.E. Dorokhov and L. Tomio, Phys.Rev. D62 (2000) 014016.

[26] I.V.Anikin, A.E.Dorokhov and L.Tomio, Phys. Lett. B475 (2000) 361, hep-ph/9909368.

[27] V.B. Rosenhaus, M.G. Ryskin Sov. J. Nucl. Phys. 44 (1986) 829 [Yad. Fiz. 44 (1986) 1276].

[28] A.R. Zhitnitsky, Phys. Lett. B329 (1994) 493, hep$\mathrm{ph} / 9401278$.

[29] N. G. Stefanis, W. Schroers and H.-Ch. Kim, Eur. Phys. J. C18 (2000) 137, hep-ph/0005218. 